\title{
Nanosomal Docetaxel Lipid Suspension
}

National Cancer Institute

\section{Source}

National Cancer Institute. Nanosomal Docetaxel Lipid Suspension. NCI Thesaurus. Code C111570.

A lipid-based nanosomal formulation of the poorly soluble, semi-synthetic, secondgeneration taxane docetaxel, with potential antineoplastic activity. Upon intravenous injection, docetaxel binds to and stabilizes tubulin, which inhibits microtubule disassembly and results in both cell cycle arrest at the G2/M phase and cell death. This liposomal formulation solubilizes docetaxel without the use of toxic solvents, such as polysorbate 80. This permits the administration of larger doses of docetaxel and improves the drug's safety profile by avoiding solvent-associated toxicities, such as hypersensitivity reactions and neurotoxicity. In addition, the nanosomal lipid-based delivery of docetaxel improves drug penetration into tumors and decreases drug clearance, all of which prolong the duration of docetaxel's therapeutic effects. 